\title{
Mappings of Nonpositively Curved Manifolds
}

\author{
By \\ Samuel I. GOLDBERG*
}

\section{§1. Introduction}

In recent papers with S. S. Chern [3] and T. Ishihara [4], the author studied both the volume- and distance-decreasing properties of harmonic mappings thereby obtaining real analogues and generalizations of the classical Schwarz-Ahlfors lemma, as well as Liouville's theorem and the little Picard theorem. The domain $M$ in the first case was the open ball with the hyperbolic metric of constant negative curvature, and the target was a negatively curved Riemannian manifold with sectional curvature bounded away from zero. In this paper, it is shown that $M$ may be taken to be any complete simply connected Riemannian manifold of nonpositive curvature. Details will appear elsewhere.

Theorem. Let $f: M \rightarrow N$ be a harmonic $K$-quasiconformal mapping of Riemannian manifolds of dimensions $m$ and $n$, respectively. If $M$ is complete and simply connected, and (a) the sectional curvatures of $M$ are nonpositive and bounded below by a negative constant $-A$, and (b) the sectional curvatures of $N$ are bounded above by the constant $-((m-1) /(k-1)) k A K^{4}, k=\min (m, n)$, then $f$ is distancedecreasing. If $m=n$ and $(b)$ is replaced by the condition $\left(b^{\prime}\right)$ the sectional curvatures of $N$ are bounded away from zero by $-A K^{4}$, then $f$ is volume-decreasing.

\section{$\S$ 2. Harmonic and $\mathbb{K}$-quasiconformal mappings}

Let $M$ and $N$ be $C^{\infty}$ Riemannian manifolds of dimensions $m$ and $n$,

Communicated by S. Nakano, October 22, 1975.

* Department of Mathematics, University of Illinois, Urbana, Illinois 61801, U.S.A.

1) This research was supported in part by the National Science Foundation. 
respectively. Let $f: M \rightarrow N$ be a $C^{\infty}$ mapping. The Riemannian metrics of $M$ and $N$ can be written locally as $d s_{M}{ }^{2}=\omega_{1}{ }^{2}+\cdots+\omega_{m}{ }^{2}$ and $d s_{N}{ }^{2}=\omega_{1}{ }^{* 2}$ $+\cdots+\omega_{n}{ }^{* 2}$, where $\omega_{i}(1 \leqq i \leqq m)$ and $\omega_{a}^{*}(1 \leqq a \leqq n)$ are linear differential forms in $M$ and $N$, respectively. The structure equations in $M$ are

$$
\begin{aligned}
& d \omega_{i}=\sum_{j} \omega_{j} \wedge \omega_{j i}, \\
& d \omega_{i j}=\sum_{j} \omega_{i k} \wedge \omega_{k j}-\frac{1}{2} \sum_{k, l} R_{i j k l} \omega_{k} \wedge \omega_{l} .
\end{aligned}
$$

Similar equations are valid in $N$ and we will denote the corresponding quantities in the same notation with asterisks. Let $f^{*} \omega_{a}{ }^{*}=\sum_{i} A_{i}{ }^{a} \omega_{i}$. Then the covariant differential of $A_{i}{ }^{a}$ is defined by

$$
D A_{i}^{a} \equiv d A_{i}^{a}+\sum_{j} A_{j}{ }^{a} \omega_{j i}+\sum_{b} A_{i}{ }^{b} \omega_{b a}^{*} \equiv \sum_{j} A_{i j}^{a} \omega_{j}
$$

with $A_{i j}^{a}=A_{j i}^{a}$. The mapping $f$ is called harmonic if $\sum_{i} A_{i i}^{a}=0$.

The differential $f_{*}$ of $f$ is extended to the mapping $\wedge^{p} f_{*}: \wedge^{p} T(M)$ $\rightarrow \wedge^{p} T(N) . \wedge^{p} f_{*}$ is also regarded as an element of $\wedge^{p} T^{*}(M) \otimes \wedge^{p} T(N)$ on which a norm is defined in terms of the metrics of $M$ and $N$. The norm $\left\|\wedge^{p} f_{*}\right\|$ is regarded as the ratio function of intermediate volume elements of $M$ and $N$. As in [4] we consider the Laplacian $\Delta$ of $\left\|f_{*}\right\|^{2}$ and obtain the following formula when $f$ is harmonic.

$$
(1 / 2) \Delta\left\|f_{*}\right\|^{2}=\sum_{a, i, j}\left(A_{i j}^{a}\right)^{2}+\sum_{a, i, j} R_{i j} A_{i}{ }^{a} A_{j}{ }^{a}-\sum_{a, b, c, d, i, j} R_{a b c d}^{*} A_{i}{ }^{a} A_{j}{ }^{b} A_{i}{ }^{c} A_{j}{ }^{d},
$$

where $R_{i j}$ is the Ricci tensor of $M$.

At each point $x \in M$, let $A$ be the matrix representation of $\left(f_{*}\right)_{x}$ relative to orthonormal bases of $T_{x}(M)$ and $T_{f(x)}(N)$ and let ${ }^{t} A$ be the transpose of $A$. In the sequel, we assume $\operatorname{rank} f_{*}=\operatorname{rank} A=k$ at every point. Then, $k \leqq \min (m, n)$ and $\operatorname{rank} G=k$, where $G$ is the positive semidefinite symmetric matrix ${ }^{t} A A$. Let $\lambda_{1} \geqq \cdots \geqq \lambda_{k}>\lambda_{k+1}=\cdots=\lambda_{m}=0$ be the eigenvalues of $G$. The norm $\left\|\wedge^{p} f_{*}\right\|$ is represented as

$$
\left\|\wedge^{p} f_{*}\right\|^{2}=\sum_{i_{1}<\cdots<i_{p}} \lambda_{i_{1}} \cdots \lambda_{i_{p}} .
$$

Lemma 1. If $k \leqq \min (m, n)$ and rank $f_{*}$ is $k$ evervwhere on $M$, then

$$
\left(\left\|\wedge^{p} f_{*}\right\|^{2} /\left(\begin{array}{c}
k \\
p
\end{array}\right)\right)^{1 / p} \geqq\left(\left\|\wedge^{q} f_{*}\right\|^{2} /\left(\begin{array}{c}
k \\
q
\end{array}\right)\right)^{1 / q}, 1 \leqq p \leqq q \leqq k
$$


At each point $x \in M$, let $S^{k-1}$ be a unit $(k-1)$-sphere in $T_{x}(M)$. If $\left(f_{*}\right)_{x}$ has maximal rank $k$, the image of $S^{k-1}$ under $\left(f_{*}\right)_{x}$ is an ellipsoid of dimension $k-1$. Let $f$ be a $C^{\infty}$ mapping of maximal rank $k$ and $K \geqq 1$. $f$ is $K$-quasiconformal if at each point $x$ of $M$, the ratio of the largest to the smallest axis of the ellipsoid $\leqq K$. One may verify that $f$ is $K$-quasiconformal if and only if $\lambda_{1} / \lambda_{k} \leqq K^{2}$ at each point. Hence, from (2) we obtain

Lemma 2. If $f$ is $K$-quasiconformal, then

$$
\left(\left\|\wedge^{p} f_{*}\right\|^{2} /\left(\begin{array}{c}
k \\
p
\end{array}\right)\right)^{1 / p} \leqq K^{2}\left(\left\|\wedge^{q} f_{*}\right\|^{2} /\left(\begin{array}{c}
k \\
q
\end{array}\right)\right)^{1 / q}, 1 \leqq p<q \leqq k
$$

\section{§ 3. Proof of Theorem}

Let $d \tilde{s}_{M}{ }^{2}$ be a Riemannian metric on $M$ conformally related to $d s_{M}{ }^{2}$. Then, there is a function $p>0$ on $M$ such that $d \widetilde{s}_{M I}{ }^{2}=p^{2} d s_{M I}{ }^{2}$. Let $\widetilde{u}=\sum\left(\tilde{A}_{i}^{a}\right)^{2}=p^{-2} \sum\left(A_{i}{ }^{a}\right)^{2}$. Then

Lemma 3. Let $f: M \rightarrow N$ be harmonic with respect to $\left(d{s_{M}}^{2}, d s_{N}{ }^{2}\right)$, and let $\widetilde{u}$ attain its maximum at $x \in M$. If the symmetric matrix function $X_{i j}=p_{i j}+\delta_{i j} \sum\left(p_{k}\right)^{2}-2 p_{i} p_{j}$, where $p_{i}$ is given by $d \log p=$ $\sum p_{i} \omega_{i}$ and $p_{i j}$ is its covariant derivative, is positive semi-definite everywhere on $M$, then $-\sum R_{a b c d}^{*} \widetilde{A}_{i}{ }^{a} \widetilde{A}_{j}{ }^{b} \widetilde{A}_{i}{ }^{c} \widetilde{A}_{j}{ }^{d} \leqq-\sum \widetilde{R}_{i j} \tilde{A}_{i}{ }^{a} \widetilde{A}_{j}{ }^{a}$ at $x$.

Let $y$ be a point of $M$ and denote by $d(x, y)$ the distance-from- $y$ function. Then, $t(x)=(d(x, y))^{2}, x \in M$, is $C^{\infty}$ and convex on $M$ (see [2]). The function $\tau(x)=d(x, y)$ is also convex, but it is only continuous on $M$. The convex open submanifolds $M_{\rho}=\{x \in M \mid t(x)<\rho\}$ of $M$ exhaust $M$, that is $M=\cup_{\rho<\infty} M_{\rho}$ (see [5]). The nonnegative function $v_{\rho}=$ $\log \frac{\rho}{\rho-t}$ is a $C^{\infty}$ convex function.

Consider the metric $d \widetilde{s}^{2}=e^{2 v_{\rho}} d s^{2}$ on $M_{\rho}$. Then $\widetilde{u}=e^{-2 v_{\rho}} u=\left(\frac{\rho-t}{\rho}\right)^{2} u$ is nonnegative and continuous on the closure $\bar{M}_{\rho}$ of $M_{\rho}$ and vanishes on $\partial M_{\rho}$. Since $\bar{M}_{\rho}$ is compact, $\widetilde{u}$ has a maximum in $M_{\rho}$. Since the function $t(x)$ is convex the matrix $X_{i j}$ is positive semi-definite, so we obtain the conclusion of Lemma 3 . 
Relating the Ricci tensors of $d \widetilde{s}_{M}^{2}$ and $d s_{M}^{2}$, we obtain

$$
\begin{aligned}
\sum \widetilde{R}_{i j} \tilde{A}_{i}{ }^{a} \tilde{A}_{j}{ }^{a}= & \left(\frac{\rho-t}{\rho}\right)^{2} \sum R_{i j} \tilde{A}_{i}{ }^{a} \tilde{A}_{j}{ }^{a}-\frac{\rho-t}{\rho^{2}}(m-2) \sum t_{i j} \tilde{A}_{i}{ }^{a} \tilde{A}_{j}{ }^{a} \\
& -\frac{\rho-t}{\rho^{2}} \Delta t\left\|f_{*}\right\|_{\rho}{ }^{2}-\frac{m-1}{\rho^{2}}\langle d t, d t\rangle\left\|f_{*}\right\|_{\rho}{ }^{2} .
\end{aligned}
$$

Lemma 4. For each $\rho$, there exists a positive constant $\varepsilon(\rho)$ such that the inequality

$$
-\sum \widetilde{R}_{i j} \widetilde{A}_{i}^{a} \widetilde{A}_{j}^{a} \leqq[(m-1) A+\varepsilon(\rho)] \widetilde{u}
$$

holds on $M_{\rho}$. Moreover $\varepsilon(\rho) \rightarrow 0$ as $\rho \rightarrow \infty$.

To see that $\Delta \tau$ is bounded as $\tau \rightarrow \infty$, observe that the level hypersurfaces of $\tau$ are spheres $S$ with $y$ as center. The hessian $D^{2} \tau$ of $\tau$ can be identified with the second fundamental form $h$ of those spheres,e xtended to be 0 in the normal direction. If follows that $\Delta \tau=$ trace $D^{2} \tau=$ trace $h$ $=(m-1) \cdot$ mean relative curvature of $S$. If the curvature $K \geqq a^{2}$, then from $[1$; pp. $247-255], \Delta \tau \leqq(m-1) a \frac{\cos a \tau}{\sin a \tau}$. If we put $a^{2}=-\alpha^{2}$, then $\Delta \tau \leqq(m-1) \alpha \operatorname{coth} \alpha \tau$.

The rest of the proof of the theorem is now a consequence of Lemmas $1-4$.

\section{References}

[1] Bishop, R. L. and Crittenden, R. J., Geometry of Manifolds, Academic Press Inc., New York, 1964.

[2] Bishop, R. L. and O'Neill, B., Manifolds of negative curvature, Trans. Amer. Math. Soc., 145 (1969), 1-49.

[3] Chern, S. S. and Goldberg, S. I., On the volume-decreasing property of a class of real harmonic mappings, Amer. J. Math., 197 (1975), 133-147.

[4] Goldberg, S. I. and Ishihara, T., Harmonic quasiconformal mappings of Riemannian manifolds, Amer. J. Math., to appear.

[5] Har'El, Z., Harmonic mappings and distortion theorems, thesis, Technion, Israel Inst. of Tech., Haifa, 1975. 